\title{
IFQuimical: uma proposta de mediação no processo ensino-aprendizagem de química
}

\author{
Janainne Nunes Alves ${ }^{1}$, Arthur Machado França de Almeida ${ }^{2}$, Marcos Daniel \\ Vieira $^{2}$, Adriano Ramos Carvalho ${ }^{2}$, Maria Alice Gomes Lopes Leite ${ }^{1}$ \\ ${ }^{1}$ Instituto Federal de Educação, Ciência e Tecnologia do Norte de Minas Gerais \\ (IFNMG) - Diamantina-MG- Brasil \\ ${ }^{2}$ Instituto Federal de Educação, Ciência e Tecnologia do Norte de Minas Gerais \\ (IFNMG) - Araçuaí-MG- Brasil \\ janainne.alves@ifnmg.edu.br, arthur.almeidadifnmg.edu.br, badamarcosdani \\ el@hotmail.com, adrianoramos20@gmail.com, alice.leite@ifnmg.edu.br
}

\begin{abstract}
The IFQuimical was developed as an interactive mediator tool in the teaching and learning process of chemistry at the secondary level, contributing to (re) signification of the curricular contents of the discipline, based on the National Curricular Parameters and the National Curricular Base of the Secondary School. This instrument provides the balancing of acidbase equations, with the respective inorganic salts formed, the images of these salts and their chemical properties, also allows the user to create simulations that are based on the National High School Examination questions of the last years, besides presenting elementary glassware available in the laboratory and its applications.
\end{abstract}

Resumo. O IFQuimical foi desenvolvido como uma ferramenta interativa mediadora no processo de ensino-aprendizagem de química, nível médio, contribuindo para (re) significação dos conteúdos curriculares da disciplina, tendo como fundamentos os Parâmetros Curriculares Nacionais e a Base Nacional Curricular do Ensino Médio. Este instrumento disponibiliza o balanceamento de equações ácido-base, com os respectivos sais inorgânicos formados, as imagens destes sais e suas propriedades químicas, também possibilita ao usuário a criação de simulados que têm como base as questões do Exame Nacional do Ensino Médio dos últimos anos, além de apresentar vidrarias elementares disponíveis num laboratório e suas aplicações.

\section{Introdução}

O processo de ensino-aprendizagem de química, como componente curricular da educação básica, envolve elementos fundamentais acerca da compreensão da constituição do mundo material, das transformações da matéria e suas relações com o dia-a-dia. Segundo os Parâmetros Curriculares Nacionais (PCN’s) elaborados pelo Ministério da Educação (MEC), que orientam sobre o ensino de química,

“[...] a química pode ser um instrumento da formação humana que amplia os horizontes culturais e a autonomia no exercício da cidadania, se o conhecimento químico for promovido como um dos meios de interpretar o mundo e intervir na realidade, se for apresentado como ciência, com seus conceitos 
VII Congresso Brasileiro de Informática na Educação (CBIE 2018)

Anais do XXIX Simpósio Brasileiro de Informática na Educação (SBIE 2018)

métodos e linguagens próprios, e como construção histórica, relacionada ao desenvolvimento tecnológico e aos muitos aspectos da vida em sociedade” [Brasil 2002].

Os PCN's referentes ao ensino de ciências da natureza, matemática e suas tecnologias (biologia, física, química e matemática), nos orientam à prática de processos de aprendizagem direcionados à produção de um conhecimento efetivo com significado próprio, desenvolvido segundo o contexto vivenciado pelos discentes, respondendo às necessidades da vida contemporânea [Brasil 2000]. As reflexões acerca das diretrizes educacionais brasileiras, apontam para o desenvolvimento de processos educacionais que contribuam para uma formação ampla, fortalecendo as bases para interpretação de fatos naturais e colaborando com a compreensão de procedimentos e equipamentos cotidianos presentes no mundo social e profissional do discente.

Esses processos educacionais buscam proporcionar ao aluno a construção de um aprendizado harmônico, cooperando com o estabelecimento de um elo sólido entre os universos escolar e social, tendo em vista que na atualidade a aprendizagem não se restringe ao ensino direcionado exclusivamente ao preparo para exames de seleção. $O$ mundo atual exige posicionamento do estudante, que este seja capaz de julgar e tomar decisões, assim como, responsabilizar-se por elas [Brasil 2002].

A complexidade do aprender requer que os educadores repensem as estratégias metodológicas que (in) viabilizam as práticas pedagógicas atuais. As mudanças no perfil do aluno, na sociedade atual, nas relações entre a escola, o cotidiano e o universo do trabalho, vinculadas aos constantes avanços científicos e tecnológicos demandam dos discentes novas competências e habilidades [Prebianca et al. 2013, Cabero 2001].

Ocorre portanto, a necessidade de novas abordagens metodológicas, o que tem resultado em um progressivo aumento na inserção de recursos tecnológicos visuais em sala de aula, entre os quais se encontram os softwares, os vídeos e os modelos moleculares, que têm se destacado na promoção de uma melhor compreensão da ciência química, em particular na educação básica [Vasconcelos e Arroio 2013, Poth 2018].

Considerando as orientações dos Parâmetros Curriculares Nacionais, as novas necessidades advindas das mudanças sociais, e inserção de novas tecnologias, o software educacional IFQuimical, foi criado com o propósito de se tornar um instrumento didático para o ensino de química, nível médio, auxiliando discentes e docentes no cotidiano escolar. O IFQuimical busca facilitar a assimilação de conteúdos através do contato com o software, que disponibiliza ferramentas vinculadas ao conteúdo curricular da disciplina, como o balanceamento de equações de neutralização ácido-base, os sais inorgânicos formados, possibilidade de criação de simulados para o ENEM (Exame Nacional do Ensino Médio). Ainda, observando a importância da experimentação e familiarização com o laboratório, o software oferece um laboratório virtual, onde se encontram as vidrarias mais utilizadas no cotidiano de um químico, suas imagens e informações sobre seu uso. Dessa forma, espera-se que o software amplie os recursos didáticos disponíveis ao professor e proporcione maior autonomia aos alunos na busca e construção do conhecimento.

O software desenvolvido apresenta essência acadêmica, e busca estimular o ensino-aprendizagem de química, através da integração entre o conteúdo curricular e as 
VII Congresso Brasileiro de Informática na Educação (CBIE 2018)

Anais do XXIX Simpósio Brasileiro de Informática na Educação (SBIE 2018)

tecnologias da informação, desenvolvendo habilidades e competências para uma aprendizagem significativa.

\section{Fundamentação teórica}

As mudanças sociais constantes, provocadas pelas tecnologias nas comunicações e informações, vêm expondo a população, em especial os jovens, a novas relações sociais e formas de interação com o mundo, assim, é construída uma nova interface de mediação e diálogo. Diante desse novo cenário social e cultural, outras habilidades são demandadas no âmbito educacional, resultando em discussões constantes a respeito de novas abordagens de estímulo à aprendizagem [Paula et al. 2012, Guaraldo e Andrade 2014].

Entre as novas metodologias e estratégias propostas para uso em sala de aula, destaca-se a implementação de materiais educativos, vistos como auxiliares em potencial para instigar o aluno, enriquecendo a aprendizagem, quando relacionados a conceitos cognitivos pré-existentes na estrutura cognitiva do educando, de modo a motivá-lo. Neste sentido, os recursos tecnológicos que já fazem parte do cotidiano dos discentes, têm se tornado alternativas criativas e dinâmicas [Cunha 2012, Chacon et al. 2013]. Esses recursos permitem um diálogo entre a comunicação e o saber, estabelecendo relações diferentes, possibilitando o uso do lúdico, informando, desafiando e oferecendo aos discentes uma pedagogia dinâmica não consciente e não diretiva [Nunes et al. 2017, Santos et al. 2017].

A recente BNCC (Base Nacional Comum Curricular) do ensino médio se mostra atenta a essa discussão, e orienta a prática de um ensino pautado na seguinte competência:

"Compreender, utilizar e criar tecnologias digitais de informação e comunicação de forma crítica, significativa, reflexiva e ética nas diversas práticas sociais (incluindo as escolares) para se comunicar, acessar e disseminar informações, produzir conhecimentos, resolver problemas e exercer protagonismo e autoria na vida pessoal e coletiva" [Brasil 2018].

As tecnologias digitais, especificamente os softwares educacionais, vêm ganhando espaço em ambientes educacionais, pela facilidade de acesso, possibilidade de abordar múltiplas linguagens, além de englobarem estímulos variados (sons, cores, movimentos) [Veletsianos 2016, Naranjo et al. 2017].

Abordagens educacionais através do uso de softwares vêm sendo compreendidas como metodologias de mediação e inclusão digital. Tendo em vista que a aprendizagem mediada constitui-se numa metodologia de ensino pautada na mediação como instrumento na ação de ensinar e aprender, onde o desenvolvimento cognitivo ocorre pela interação do indivíduo com o meio que o cerca [Ferreira et al. 2015].

Para Vygostky (1996) e Fuerteins (1994, 1997), citados por [Prebianca et al. 2013], o aprender ocorre pela interação do indivíduo com o meio, processo que possibilita a maturação biológica das estruturas cognitivas do ser humano, através da interação com instrumentos que ampliam a visão de mundo. Piaget (1999), também faz menção à aprendizagem pela interação com o objeto, onde o desenvolvimento do indivíduo se dá em etapas, através da construção de esquemas mentais, que são reelaborados pela interação com o objeto. A construção de esquemas implica numa assimilação e acomodação, modificando a forma de pensar [Paula et al. 2012]. 
VII Congresso Brasileiro de Informática na Educação (CBIE 2018)

Anais do XXIX Simpósio Brasileiro de Informática na Educação (SBIE 2018)

A mediação permite que valores socioculturais, não disponíveis ao aprendiz pela interação direta do indivíduo com o meio, sejam transmitidas a ele. E dessa forma, corrobora com a reestruturação dos padrões cognitivos adquiridos. Neste contexto, os softwares educacionais como mediadores de aprendizagem, podem convidar os alunos/usuários a transcender seu estágio de conhecimento atual, solucionando problemas cada vez mais complexos [Prebianca et al. 2013].

Além da possibilidade de atuação como instrumentos de mediação, a inclusão de novas tecnologias na educação, tem revolucionado os processos de ensinoaprendizagem, ampliando o acesso aos diferentes recursos didáticos e minimizando diferenças regionais e de infraestrutura, muitas vezes encontradas nestes ambientes [Bralié e Divjak 2018, Hultquist 2016].

\section{Metodologia/desenvolvimento}

O software, IFQuimical, foi projetado como auxiliar no processo ensino-aprendizagem, relacionado à disciplina da química. Seu desenvolvimento ocorreu em etapas, onde no primeiro momento, foram levantadas as funcionalidades do sistema destinadas a apoiar discentes e docentes no ensino-aprendizagem da disciplina. Analisados os graus de dificuldade relacionados à aprendizagem, foram propostas as seguintes funcionalidades: balanceamento de equações ácido-base, simulado de química com questões do ENEM e familiarização com os materiais presentes num laboratório de química.

Num Segundo momento, foi realizada uma busca por informações para construção da base de dados do sistema. Nessa etapa foram investigados dados sobre as reações ácido-base, os reagentes necessários, os sais inorgânicos produtos das reações de neutralização, assim como, as propriedades essenciais destes sais. Realizou-se ainda o download das avaliações do ENEM referentes aos últimos dez anos, e os conteúdos da disciplina química, foram categorizados de acordo com a organização curricular do ensino médio. As principais vidrarias de laboratório presentes no cotidiano de um químico, assim como, suas principais aplicações, também foram selecionadas.

Paralelamente ao levantamento das informações para compor a base de dados do sistema, foram elencadas as ferramentas necessárias ao desenvolvimento da proposta. Para construção do sistema, foram definidas algumas bibliotecas livres disponíveis na internet: Codeigniter, framework PHP baseado no padrão MVC (ModelViewController); Jquery, framework Javascript; Bootstrap, framework para construção de interfaces responsivas que utiliza CSS e Javascript; além de HTML 5 para construção das interfaces.

O SGBD (Sistema de Gerenciamento de Banco de Dados) escolhido foi o MySQL, que é relacional e utiliza a linguagem SQL para manipulação dos dados. Para manipulação dos dados do MySQL, foi utilizada a ferramenta MySQL Workbench. Já a linguagem de programação escolhida para o desenvolvimento do software foi o PHP 7.0.30. A seleção das ferramentas mencionadas, teve como premissa essencialmente a facilidade de aplicação, que tem reflexos diretos sobre a curva de aprendizagem. Fatores relevantes, visto que, na elaboração, criação e desenvolvimento do sistema, foram envolvidos alunos do Instituto Federal de Educação, Ciência e Tecnologia (IFNMG) do campus Araçuaí. O terceiro momento de construção do IFQuimical, foi dedicado ao desenvolvimento e modelagem da base de dados. Foi elaborado o Diagrama de Casos de 
VII Congresso Brasileiro de Informática na Educação (CBIE 2018)

Anais do XXIX Simpósio Brasileiro de Informática na Educação (SBIE 2018)

Uso do Sistema e o Modelo Entidade-Relacionamento da base de dados. Nessa etapa, todas as informações relacionadas foram inseridas na base de dados, por meio da execução de scripts SQL no MySQL Workbench.

Finalizadas as etapas anteriores, foi iniciado o desenvolvimento do código, utilizando as ferramentas anteriormente definidas para inserção das funcionalidades previamente selecionadas. Com o propósito de gerenciamento do software, foi desenvolvido também um módulo de administrador do sistema.

No módulo de administrador do software, o usuário consegue realizar o cadastro e edição de questões e materiais presentes no sistema, possibilitando novas implementações e atualizações. $O$ acesso ao módulo de administrador é realizado exclusivamente por meio de login e senha, sendo inacessível a usuários que não possuem as credenciais de acesso.

O software foi elaborado de forma a englobar funcionalidades distintas, no entanto, todas estas se relacionam ao ensino da química, nível médio. Um diagrama de casos de uso do IFQuimical é apresentado na figura 1, e são definidos esquematicamente os atores e funcionalidades do sistema desenvolvido.

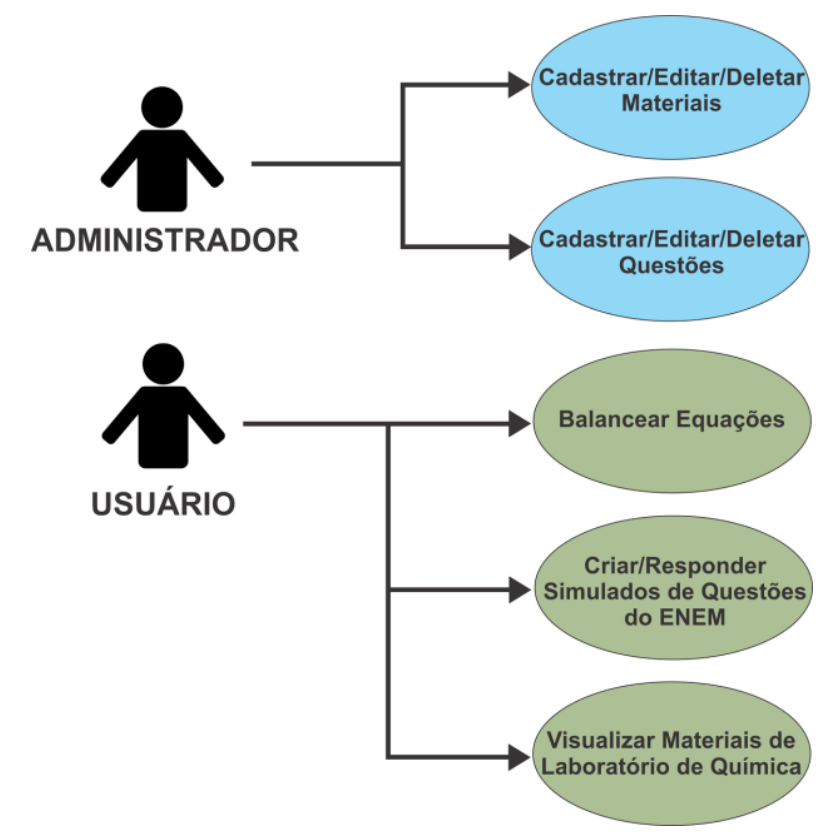

Figura 1. Diagrama de Casos de Uso do IFQuimical

\section{Apresentação do software}

A interface inicial do IFQuimical é apresentada na figura 2, onde é possível observar as três funcionalidades abordadas pelo sistema. As funcionalidades serão detalhadas nas próximas subseções. 
VII Congresso Brasileiro de Informática na Educação (CBIE 2018)

Anais do XXIX Simpósio Brasileiro de Informática na Educação (SBIE 2018)

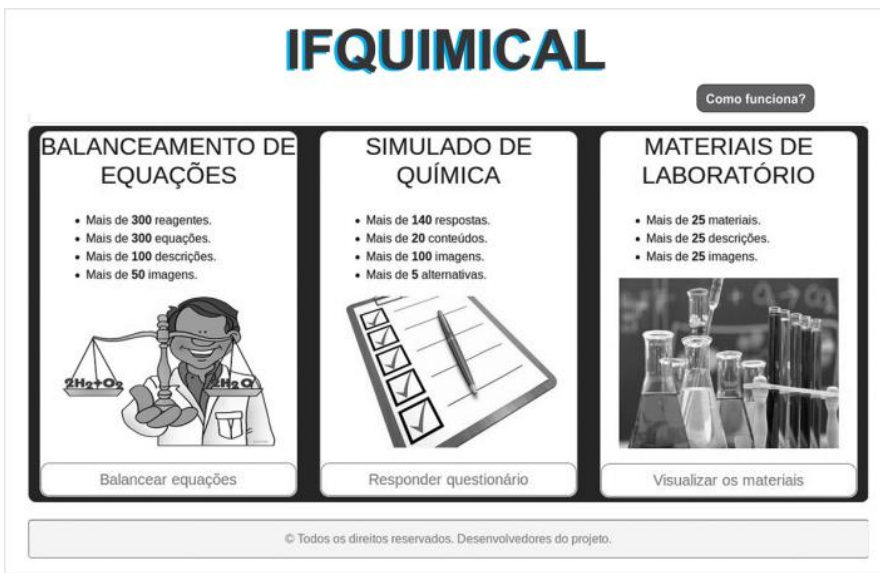

Figura 2. Tela inicial do IFQuimical

\subsection{Balanceamento de equações químicas}

A funcionalidade "Balanceamento de equações químicas" aborda aspectos relacionados às equações de neutralização ácido-base. $\mathrm{O}$ usuário do software insere a equação de balanceamento e o sal inorgânico, produto da equação de neutralização selecionada, é apresentado com sua imagem e informações elementares (nome, suas temperaturas de fusão e ebulição, massa molar, densidade e aplicações).

Na figura 3A, é possível observar a tela onde são disponibilizados os campos para seleção dos reagentes e balanceamento da equação. Na sequência, a figura $3 \mathrm{~B}$, traz o sal resultante do balanceamento e suas propriedades químicas.

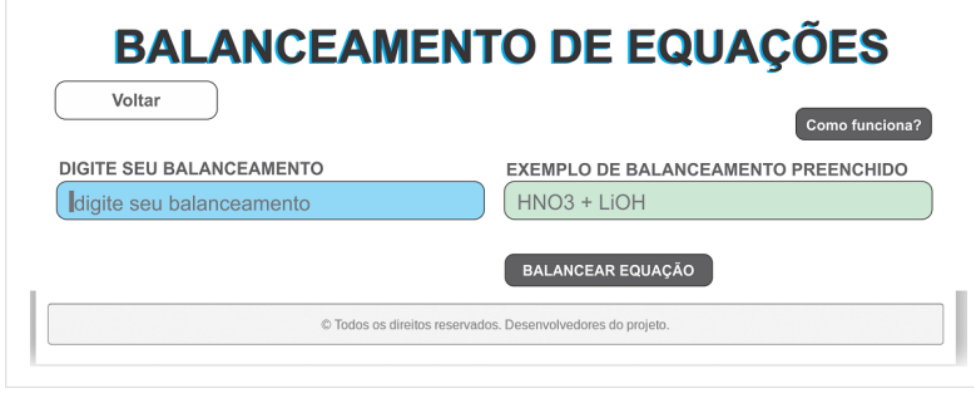

(A)

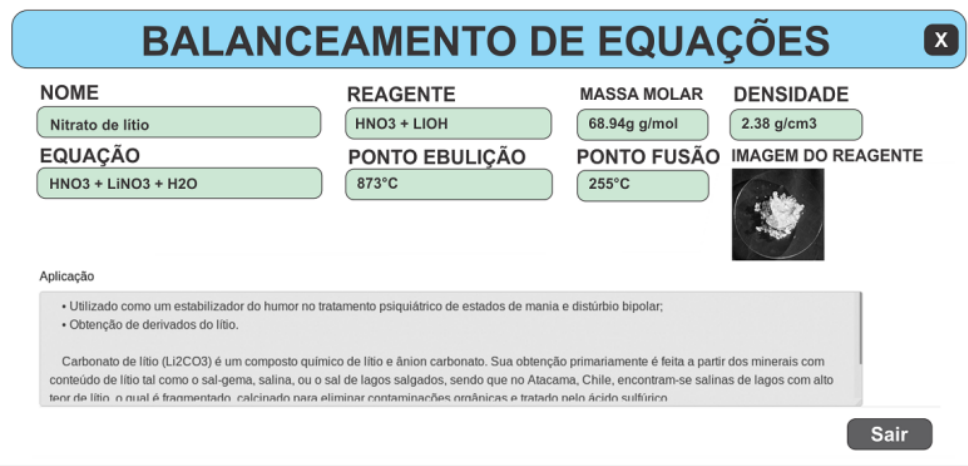

(B)

Figura 3. (A) Seleção dos reagentes e equação de neutralização ácido-base; (B) Sal inorgânico formado e suas propriedades 
VII Congresso Brasileiro de Informática na Educação (CBIE 2018)

Anais do XXIX Simpósio Brasileiro de Informática na Educação (SBIE 2018)

\subsection{Simulado com questões do ENEM}

No "Simulado com questões do Enem" o usuário tem acesso às questões do Enem, aplicadas nos últimos dez anos do exame, relacionadas ao conteúdo programático de química. A criação deste tópico se deu através do agrupamento das questões nos seguintes tópicos: Acidez e Basicidade, Concentração, Densidade, Eletroquímica, Equilíbrio Químico, Estequiometria, Fenômeno Físico e Químico, Forças Intermoleculares, Funções Inorgânicas, Funções Orgânicas, Polaridade e Solubilidade, Propriedades Coligativas, Química Orgânica, Química Verde, Radioatividade, Reações Inorgânicas, Soluções, Substâncias e Misturas, Termoquímica e Transformações Químicas.

Para construção do simulado, o usuário deve selecionar os tópicos desejados (é possível selecionar mais de um tópico) e o software sorteia dentre as questões presentes na base de dados, aquelas que o usuário irá responder, o que possibilita a resolução e confecção de inúmeros simulados. Após o preenchimento do questionário, o usuário submete suas respostas e o software retorna o resultado do simulado.

No resultado, são destacadas todas as questões que foram respondidas corretamente e as que apresentaram erro. $\mathrm{O}$ usuário pode ainda, visualizar o texto da questão, para fazer uma revisão. Na figura 4 é possível observar detalhadamente essa funcionalidade.

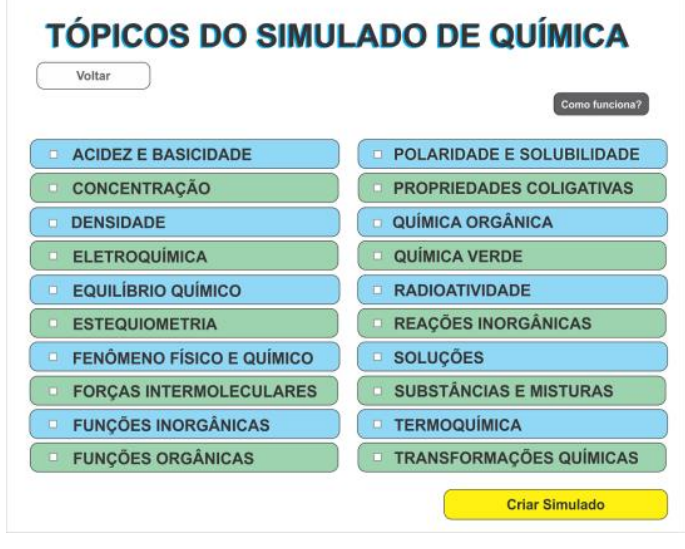

(A)

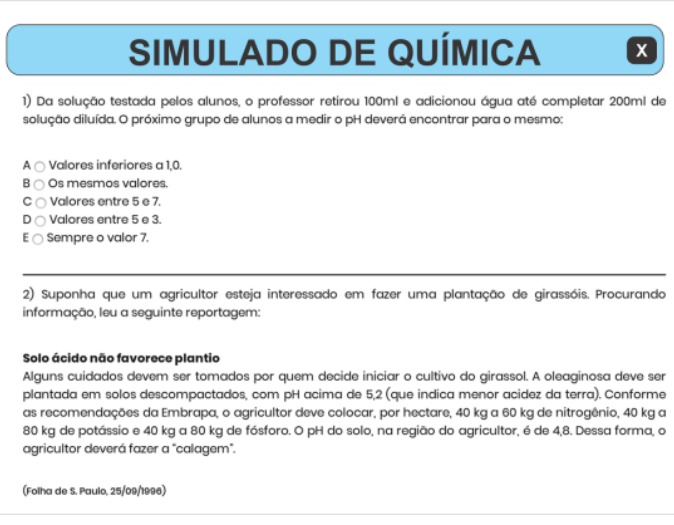

(B)

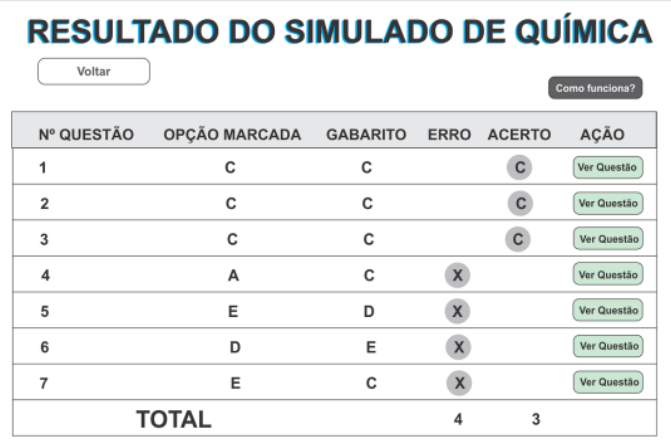

(C)

Figura 4. (A) Tópicos disponíveis para elaboração do simulado; (B) Apresentação do simulado; (C) Gabarito e total de pontos alcançados pelo usuário. 
VII Congresso Brasileiro de Informática na Educação (CBIE 2018)

Anais do XXIX Simpósio Brasileiro de Informática na Educação (SBIE 2018)

\subsection{Demonstrações de materiais utilizados em laboratórios de química}

A presente funcionalidade busca apresentar ao usuário os materiais (vidrarias) que são comumente utilizados nos laboratórios de química, se propondo a minimizar a distância entre o aluno e o universo experimental. Para sua execução, o usuário seleciona uma vidraria que se encontra em uma lista, e então, a imagem do material selecionado, assim como, suas informações essenciais (nome e descrição elementar sobre seu uso em laboratório) podem ser acessados. Nas figuras 5 A e 5B, são apresentadas as telas disponibilizadas ao usuário neste laboratório virtual.

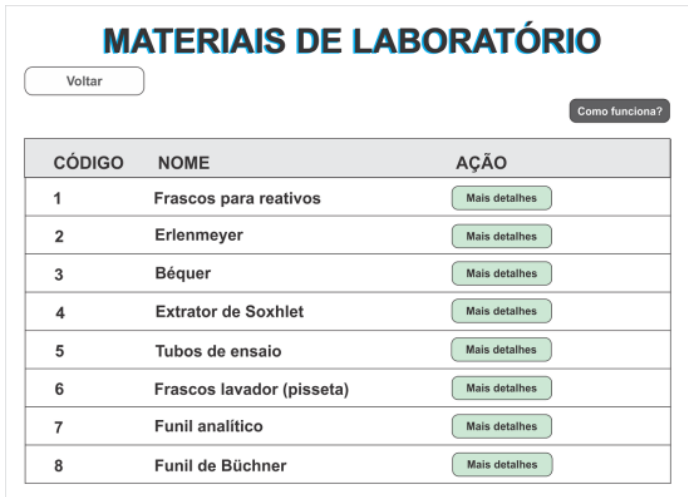

(A)

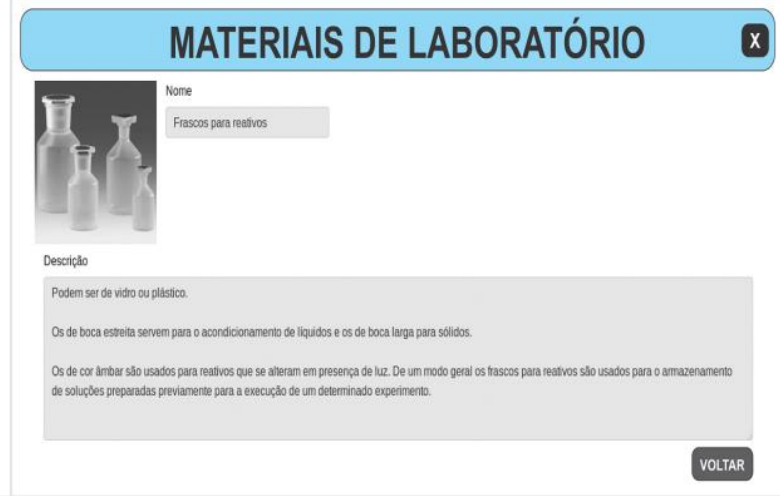

(B)

Figura 5. (A) Seleção das vidrarias; (B) Imagem e informações acerca da vidraria selecionada.

Diferentemente dos simuladores online para balanceamento de equações e laboratórios virtuais atualmente disponibilizados na internet, o IFQuimical traz imagens $\mathrm{e}$ informações sobre os sais inorgânicos gerados e reúne outras duas funcionalidades (simulado do ENEM e apresentação de vidrarias). A facilidade em elaborar simulados com questões específicas contidas no ENEM pela divisão das questões de acordo com tópicos curriculares da disciplina, permite ao usuário se aprofundar em conteúdos específicos. O modo de sorteio das questões selecionadas pelo usuário, quanto ao conteúdo, permite que sejam gerados inúmeros simulados diferentes, ampliando as possibilidades de estudo. De forma a contribuir com o preparo do estudante que tem como objetivo o ingresso em instituições de ensino superior ou mesmo, estudos diários, tendo em vista uma tendência das instituições em aceitar o ENEM como pré-requisito para ingresso. Já as imagens das vidrarias, assim como, sua aplicação no laboratório, também incorporadas ao software, proporcionam a familiarização do estudante com o laboratório. A inserção da última funcionalidade mencionada, ocorreu pela deficiência ou mesmo ausência de infraestrutura laboratorial na maioria das escolas brasileiras. Outro diferencial do software é a união de aplicações distintas num mesmo sistema, otimizando a busca e ampliando o espectro de conteúdo acessível ao usuário.

\section{Considerações finais}

O IFQuimical, foi construído para uso como plataforma interativa, tratando-se de um recurso midiático que tem como objetivo mediar a aprendizagem de química no ensino médio, contribuindo para (re) significação dos conteúdos curriculares da disciplina, tendo como fundamentos os Parâmetros Curriculares Nacionais e a Base Nacional 
VII Congresso Brasileiro de Informática na Educação (CBIE 2018)

Anais do XXIX Simpósio Brasileiro de Informática na Educação (SBIE 2018)

Curricular do ensino médio. O uso do software amplia os recursos didáticos disponíveis nas salas de aula do ensino médio, possibilitando uma aprendizagem significativa, com o auxílio de tecnologias computacionais.

O presente software foi desenvolvido no Instituto Federal de Educação, Ciência e Tecnologia do Norte de Minas Gerais (IFNMG), de modo que vislumbra-se num primeiro momento disponibilizar o software aos discentes e docentes de todos os campi da instituição. Num segundo momento será realizada uma análise da exequibilidade do software em sala de aula, através da coleta de dados resultantes de sua aplicação e uso pelos docentes e discentes do IFNMG. Posteriormente à análise, sua disponibilização será ampliada para uso em instituições de ensino médio, das redes federal, estadual e privada.

Quanto aos melhoramentos e novas implementações, pretende-se liberar ao aluno/usuário, vídeos curtos com experimentos fundamentais realizados em laboratório, além da disponibilização de um simulado no formato "jogo de tabuleiro", como forma de introduzir ludicidade e tornar o estudo mais divertido. Também será desenvolvida a versão mobile (Android) do software, permitindo aos estudantes baixar o aplicativo para uso no smartphone, ampliando suas formas de acesso.

\section{Referências}

Bralić, A., \& Divjak, B. (2018) "Integrating MOOCs in traditionally taught courses: achieving learning outcomes with blended learning", International Journal of Educational Technology in Higher Education, p.2-16.

Brasil. (2000) "Parâmetros Curriculares Nacionais para o Ensino Médio". http://portal.mec.gov.br/seb/arquivos/pdf/ciencian.pdf, junho.

Brasil. (2002), "Parâmetros Curriculares Nacionais para o Ensino de Química". http://portal.mec.gov.br/seb/arquivos/pdf/09Quimica.pdf,junho.

Brasil. (2018) Base Nacional Comum Curricular do Ensino Médio. http://basenacionalcomum.mec.gov.br/wp-content/uploads/2018/04/BNCCEnsino Medioembaixasite.pdf, junho.

Cabero, J. (2001) "Tecnología educativa. Diseño y utilización de medios en la enseñanza”, Docencia Universitaria, p.97-99.

Chacon, E. P., Ribeiro, C. M. R., \& Borges, M. N. (2013) "Comunicação química elaboração de um software educativo para motivar a aprendizagem da química orgânica", Enseñanza de las ciencias, (Extra), 2688-2694.

Cunha, M. B. (2012) "Jogos no ensino de química: considerações teóricas para sua utilização em sala de aula", Química Nova na Escola, São Paulo,p. 92-98.

Ferreira, A. C. D. L., Vilas-Boas, T. D. J. R., Silva, A. B. D. S. M., de Souza Araújo, T., dos Santos, L. M., \& de Souza, D. B. (2015) "Formação do professor mediador: inclusão e intervenção psicopedagógicas", Revista de Estudios e Investigación en Psicología y Educación, p. 238-241. 
VII Congresso Brasileiro de Informática na Educação (CBIE 2018)

Anais do XXIX Simpósio Brasileiro de Informática na Educação (SBIE 2018)

Guaraldo, F., Andrade, A. C. (2014) "Fatores humanos na avaliação e design de interfaces digitais educativas para crianças em idade escolar", Blucher Design Proceedings, p.3722-3733.

Hultquist, B. L. (2016) "Innovative teaching strategies in nursing and related health professions", Jones \& Bartlett Publishers p.1-625.

Naranjo, C. A., Ortiz, J. S., Álvarez, V. M., Sánchez, J. S., Tamayo, V. M., Acosta, F. A., ... \& Andaluz, V. H. (2017) "Teaching Process for Children with Autism in Virtual Reality Environments", In Proceedings of the 2017 9th International Conference on Education Technology and Computers p. 41-45.

Nunes, B. P., Martins, I., de Campos, G. H. B., Heinsfeld, B., Cherman, A., \& Haun, L. G. (2017), "iPlanetário Da Terra ao Universo", In Anais dos Workshops do Congresso Brasileiro de Informática na Educação,p.192-199.

Paula, D. G., Santos, A. C. O., \& de Castro, G. A. S. (2012) "Aprendizagem mediada pelo computador: as crianças e as telas digitais", Revista Tecer, p.53-66.

Piaget, J. (1999) "The stages of the intellectual development of the child, The Blackwell in development psychology, p. 26-35.

Poth, C. (2018) "The contributions of mixed insights to advancing technology-enhanced formative assessments within higher education learning environments: an illustrative example", International Journal of Educational Technology in Higher Education, p.919.

Prebianca, G. V. V., dos Santos Junior, V. P., Momm, C. F., da Silva, L. F., \& Nehring, H. (2013) "O uso de softwares educacionais como ferramentas mediacionais e de inclusão tecnológica”, ETD: Educaçao Temática Digital, 15(3), 474-494.

Santos, W. H. L., Del Pino, J. C., Sá-SIilva, J. R., \& Pinheiro, R. S. (2017) “A ideia do lúdico como opção metodológica no ensino de ciências e biologia: o que dizem os tcc dos egressos do curso de ciências biológicas licenciatura da universidade federal do rio grande do sul?”, Pesquisa em Foco, p.176-194.

Vasconcelos, F. C. G. C., \& Arroio, A. (2013) "Explorando as percepções de professores em serviço sobre as visualizações no ensino de química", Quim. Nova, p.1242-1247.

Veletsianos, G. (2016) "The defining characteristics of emerging technologies and emerging practices in digital education", Emergence and innovation in digital learning: Foundations and applications, p.3-16. 